\title{
Design of An Intelligent Heat Supply Monitoring System based on Android System
}

\author{
Luoya Guo \\ School of Information Engineering, Xi'an University,710065
}

Keywords: Intelligent heating, Android system, Database, Monitoring platform

\begin{abstract}
In recent years, most cities have realized urban central heating, but also built their own intelligent heating networks. However, there are still many problems in the control and management of heating network. In this paper, through the advanced Android system, an intelligent heating monitoring system is established, which realizes the combination of the monitoring platform, the heating equipment and the intelligent terminal. With the Android smart phone as the system terminal, the real-time monitoring of heating is carried out, and the client users can monitor the heating condition in real time. The new management method is applied to the actual heating monitoring, to achieve a design of intelligent heating monitoring system based on Android system, improve work efficiency and save energy.

With the application of smart heating system, many cities nowadays have achieved centralized urban heating, and the establishment of intelligent heating system makes the control of heating system more integrated ${ }^{[1-5]}$. In recent years, the strategy of "Internet + " has been gradually implemented in all fields. The new management of big data, which is based on the idea of "Internet $+"$, is also gradually well-known. To achieve intelligent, information management, can effectively optimize the structure of enterprises, enhance the efficiency of enterprises. Fine management has gradually been recognized, widely used in various fields ${ }^{[6-10]}$.

In this paper, in order to bring the idea of big data into the heating monitoring system, an intelligent heating monitoring system based on Android system is proposed. Android APP is designed for real-time monitoring of heating and system inspection, timely collection, convenient front-line heating staff to control the heating system, improve work efficiency.
\end{abstract}

\section{Data Base of Heat Supply Monitoring System}

The application of intelligent terminal Android mobile phone to the central heating management is based on the policy guidance of the quantitative management reform so as to be close to the actual demand. Automatic control of thermal station, quantitative heat management and application of intelligent terminal application is the "initial" and "end" relationship, the combination of the two can form the intelligent management of big data. Therefore, before the application content of the intelligent terminal is elaborated, this article first analyzes the basic content on which it depends.

Management mode

Before the heat supply monitoring system is established, all the thermal stations have adopted the fine operation management model of "one station one day one plan". This management mode is based on thermal management platform, build a heating area heating station, heat index, meteorological temperature and heating demand of the model, collected a total of three years of thermal power station energy consumption data, energy consumption of each station through these data and heat were analyzed by heat, and heat users the thermal characteristics of the fine management of heating demand, reducing cost, saving energy and reducing consumption.

Through the "one station one day one plan", the initial quantitative management of the thermal power station is completed, which makes the basis of heating production change. This management mode is used to build the foundation for the later technology.

Unattended system

Like the "one station one day one plan" management mode, the unattended system is also the foundation for establishing the data for the intelligent heating monitoring system. The data 
information collected by the system is the data source of all the functions of intelligent terminal Android mobile APP.

Before using the unattended system, the thermal power station has always used the original thermal monitoring system. There are some problems in the original thermal monitoring system, which can generally be generalized into three aspects.

The original thermal monitoring system can only monitor data of primary entrance and lack other parameters, so that the thermal power station can not fully understand the actual operation status.

only one time the total port flow limiter with electric actuator can be adjusted and controlled, lack of user and subsystem control function. For these single - channel standing stations, there is no way to regulate the system through the heat required by the two line.

In the communication of data, the methods used are very confusing, including CDMA dynamic address, radio and fixed IP. This will bring unnecessary delay to the monitoring system of the whole thermal power station.

The unattended system is a large-scale upgrading of the original monitoring equipment as a whole. At the same time, all the independent control units in the thermal power station are integrated to solve some problems existing in the original monitoring system. The unattended system is divided into two parts from the structure: the data platform system and thermal power station monitoring system, the function of the data platform is based on the so-called original monitoring platform, to improve the data acquisition, add two parameters and the corresponding data management function; transformation equipment is the old control system as the basis, using some of the more advanced monitoring technology and network technology, the thermal station data uploaded to the management platform, can remotely control the parameters of the upper platform issued by the receiving station, a thermodynamic parameter control, fault alert and abnormal warning function. The communication between the host computer and the monitoring system is the use of VPN line and 3G telecommunications network, fixed by IP, using the Modbus protocol, according to the Lord from the structure, the establishment of a data platform and monitoring equipment of substation communication links, can receive various kinds of data.

\section{System Demand Analysis}

The use of unattended monitoring equipment and data platform can realize the remote control of each management layer to the thermal station. In order to further extend the use value of the monitored data, letting the information can be obtained by front-line employees. The research and development of the remote intelligent heating monitoring system based on the intelligent terminal equipment of android system is carried out in this paper. According to the operation experience of the unattended platform in three years and the operation research of relevant heating, the actual requirements for the mobile terminal android application are summarized in the following three aspects:

\section{Holistic scheme}

For intelligent terminals, the source of data source should be the data collected by monitoring devices of the existing unattended monitoring platform, and no need to add new devices to collect data, which will save a lot of cost, meanwhile without adding new devices, the data of the intelligent terminal can be consistent with the data of the existing management platform. The intelligent terminal is connected to the unattended system, which can effectively implement the extension of the existing management platform.

\section{Economic and practical}

When designing the remote intelligent monitoring system for mobile terminals, we need to consider the employees. Because most of the front-line heating staff use Android phones, so in order to save the cost of terminal purchase, we design Android smart phone to install APP.

\section{Simple and easy to use}

For the technical personnel of the thermal station, the function of the intelligent terminal remote monitoring APP is to facilitate the adjustment and use of the equipment and the parameters, 
therefore, the interface of terminal should be easy to understand, and the operation mode of the whole interface should be basically consistent with the actual field equipment and operation platform, so that it is convenient for technicians to operate.

So from the above analysis it can be concluded that the development principle of intelligent terminal APP should be: using low construction cost, enhance the monitoring equipment installation return; using intelligent mobile phone as a smart terminal hardware platform, realize the intelligent monitoring function is applied to the mobile phone; Connecting the public network with the upper server, giving full play to the usability of the monitoring system, optimizing the management structure of the data, and realizing the direct acquisition of the monitoring data by technicians.

\section{System Architecture Design}

\section{System architecture}

First, the intelligent terminal Android APP takes the data obtained from the unattended data monitoring platform as the database, configuring a database server, providing the data access service for the intelligent terminal Android APP, and building a database cluster with the server of the unattended platform, transferring data to all monitoring platforms, the intelligent terminal APP reads the data and forms a new real - time, historical data record on the terminal. The static data based on APP is stored in the intelligent terminal.

Through the above analysis, we can know that the data obtained by smart phone APP are all based on the unattended monitoring platform, so the unattended monitoring platform is the database of intelligent terminals. It is therefore necessary to configure a database server to provide data access services to the intelligent terminal.

\section{Introduction of Android application}

The main function of APP is the real-time monitoring function. This function is to inquire and analyze all the information of the thermal power station, and to understand the real-time operation status of the thermal power station. This function must set a certain jurisdiction scope. The real-time monitoring function includes all branch systems and the inlet of the thermal power station. The thermal station involved is indirect heating, so when monitoring the heating station of the heating system, the main monitoring contents are:

A thermal station primary entrance parameters, such as pressure, temperature, and flow. According to the monitoring of the temperature and pressure parameters of the primary network, the system can master the actual heating condition and adjust it in time through the current situation.

The opening parameters of the regulating valve of each branch system and the one time temperature parameter. The effectiveness of the actuator opening and the automatic control of each branch is determined according to the monitoring of each actuator and the one back temperature of each branch.

The water supply and return pressure of the two pipeline and the temperature parameters. The temperature of the two water supply and return water is monitored to observe whether the opening degree of the one branch actuator is reasonable and whether the temperature of the heat medium reaches the standard. The pressure of the two water supply and return water is monitored and the running condition of the circulating pump is determined.

All data of the branch system are polling based on a certain period of time for the local monitoring equipment. The period is 12 minutes, which is based on the heating system as the system characteristics of process control, ensuring the real-time performance of all monitoring data. The data collected are stored in the server, waiting for the access of the intelligent terminal.

In addition to the above main monitoring contents, the intelligent terminal Android APP designed in this paper can also analyze the running state of the thermal power station based on the collection of all the logs in the server, the information of the log contains five kinds of data: alarm record, control log, single consumption query, district heating area complaint rate, central heating geographic information system.

Alarm record 
The alarm inquiring can be realized through APP, and the alarm information of the monitoring platform can be learned, and what time the problems have occurred in which parameters.

Control log

It can carry out the unattended system equipment control way record the query, to the site all real-time operation information to understand, the heating condition of the judgment.

\section{Single consumption query}

This feature enables a technician to make a query about the heat loss at a certain heat station. This function can be used to understand the heat situation of the heat station, including daily heat and daily heat supply. According to the query of historical information, it is possible to judge the operation of the heating condition, whether the planned heating indexes of "one station one day one plan" are reached, whether the heating quantity needs to be adjusted.

\section{The rate of district complaints in heating}

The heating area complaint information and the heating monitoring system are connected to the heating station, and then the information is sent to the intelligent terminal. The heating regulator can learn the heating problems timely through the information release of the intelligent terminals, and solve the effective problems in time.

\section{Geographic information system for central heating}

Merging geographic information with transmission and distribution pipeline information, real-time positioning, navigation system, etc, is good for the heat mediators to understand the current state of the pipeline, mastering the heat source distribution mode of the primary heat supply network in the city, and to strengthen the transparency of the first heat network, so that the distribution of the distribution pipeline is more reasonable. In addition, it can also provide route for the repair personnel of the heating system by navigation, and avoid the delay of repair due to the path problem.

\section{The Realization of the System Function}

The structure system of the whole intelligent heating monitoring system adopts the C/S mode, it can be seen from the following diagram that the intelligent heating monitoring system is divided into three parts: the embedded system based on the controller area network CAN bus, the heating monitoring system and the intelligent terminal Android APP. Each subsystem is independent, and the heating monitoring system is the middle part of the system. interacting with the heating monitoring system and the intelligent terminal android APP, has low dependence on the aggregate, and it can be extendibility. The following mainly introduces the collection and monitoring of energy consumption information, the data display and control of the heating flow of the client, and how the two system functions are realized.

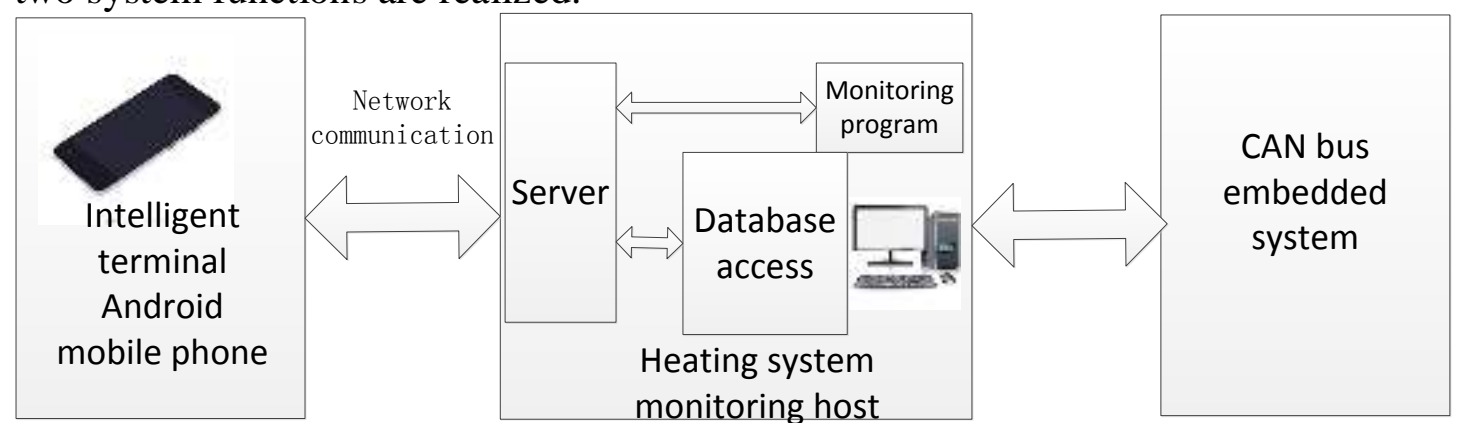

Figure 4.1. General schematic diagram of intelligent heating monitoring system

\section{Energy consumption information collection and monitoring}

Embedded system based on CAN bus is responsible for the heating system of data acquisition, such as the temperature of the heating system parameters, pressure and heat flow parameters and so on, but also controls the corresponding actuator, the actuator of the opening control the heating system the change of physical parameters.

In this paper, the SHCAN2000 field bus control system of the three Dalian instrument company is used to communicate with the upper computer of the heating monitoring platform, realizing the 
information acquisition and instruction control of each heat network unit is realized. For example, temperature information is collected through the field measurement of Pt100 and the signal CAN be sent to the CAN field bus system. CAN field bus system sends the collected data to the heating system and receives the control order of the heating system. The monitoring host of the heating system is mainly responsible for the monitoring of the heating site and the data processing of the related parameters. After processing the data, the monitoring master is able to store the collected data and then send it to the intelligent terminal APP by the corresponding server. Intelligent terminals use mobile networks and servers to communicate, and front-line heating staff can implement parameter data and device information query, device control and other functions on intelligent terminal APP.

\section{Data display and control of the heating flow of the client}

The intelligent terminal Android APP needs to query the SQL Serve database when sending the login request or inquiring the status information request of the heating system. In general, front-line heating staff is only concerned with the data on the intelligent terminal APP, and does not care about how the data is stored and where it exists. In the intelligent heating monitoring system designed in this paper, the SQL data access method is used to shield the type of the database source, and only to focus on the use of data. When front-line heating staff use the intelligent terminal APP to send requests for a parameter data to the server, the request includes the information of the logged users and the information that users want to operate on the data. After the server receives the user's request, first step would be to validate user login permissions, validation is done, if passed, would start to perform the requested operation, through to the database query to find the corresponding data table, the server program the SQL statement parsing, then will get the information the user wants to return to the user. The overall design of data access is shown below.

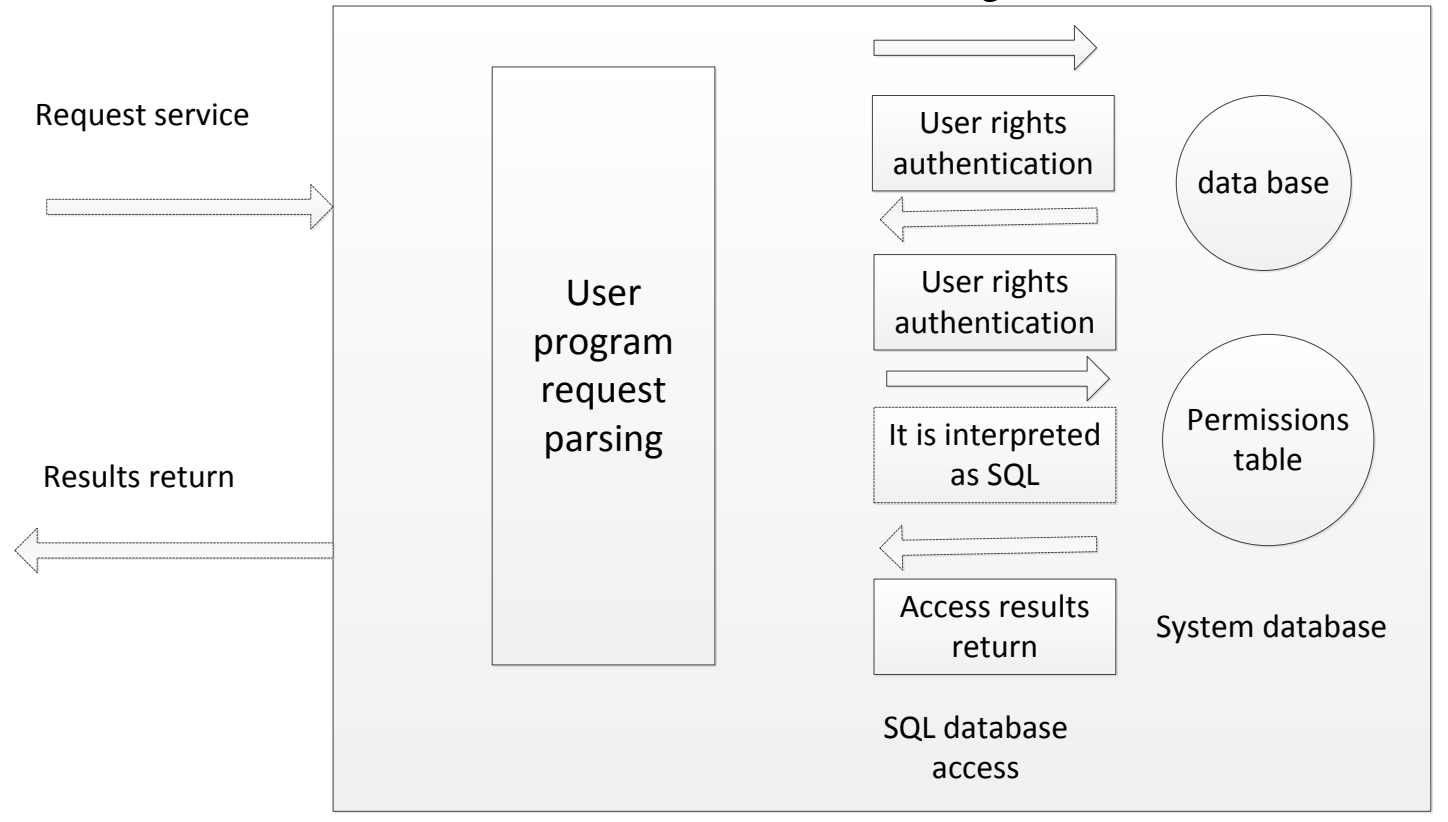

Figure 4.2. Server access database schematic

After receiving the corresponding data on the intelligent terminal Android APP, the front-line heating staff will make the control decision making through the analysis and judgement of the data, and send the control instructions to the monitoring host through the server. Monitor host gets APP back data and control instructions from the server, storing these data in the SQL database of the host, monitor host controls the data through the returned data and the control instructions, the control instruction is transferred to the equipment layer of the heating site through the embedded system, and then the control action of the control instruction is completed.

An example of the process of data acquisition and control with heating flow, first, in the field of heating, the embedded system based on CAN bus will measure the heating flow through the corresponding flow sensor, and collect the traffic data into the system, the data will be processed in the heating monitoring system, after processing, the monitoring host will store the data and send the 
corresponding server to the intelligent terminal APP. At this time, front-line heating staff can get the heating flow of the heating site through the intelligent terminal APP, if the front-line employees want to adjust the heating flow, then they can operate on the APP interface, APP sends the control instructions to the monitoring host of the heating system through the server. The monitoring host gets the control instruction, and the control instruction is passed to the equipment layer of the heating field through the embedded system, that is, the flow control valve at the scene, the valve acts, and then completes the control of the heating flow.

\section{Conclusion}

In this paper, through the advanced Android system, an intelligent heating monitoring system is established, which realizes the combination of the monitoring platform, the heating equipment and the intelligent terminal. With the Android smart phone as the system terminal, the real-time monitoring of heating is carried out, and the client users can monitor the heating condition in real time.

\section{Acknowledgements}

Fund Project: Xi'an science and technology planning project (No.2017CGWL37).

\section{Reference}

[1] Dong Hong-wei.Software and Hardware Design on Intelligent Heating Monitoring System[J].Industrial Technology Innovation, 2017,04(03):36-38.

[2] Xie Mu-jun, FENG Jing-fang, JIANG Chang-Hong.Research of Secondary Network Backwater Temperature Forecast and Control for Centralized Heat-supply System[J].Control Engineering of China, 2015,22(02):291-295.

[3] Feng Yi-wu,FEI Pan-feng.Research and Application of Internet + Intelligent Central Heating System[J].Power Generation \& Air Condition,2016,37(05):74-78.

[4] Yang Tian-wen.Application of automation technology in urban central heating[J].HEILONGJIANG SCIENCE,2016,7(16):46-47.

[5] Jiang Qianqian, FANG Xiumu,JIANG Yongcheng,ZHOU Zhigang,etc.Basic Research on Intelligent Heat-supply and Its Informatization Architecture[J].GAS \& HEAT,2015,35(02):26-30.

[6] Liang Tao,XU Guannan, YANG Weida,ZOU Jihang.An Intelligent Information Acquisition System for Heat Exchange Station Based on LBS[J].Journal of South-Central University for Nationalities( Nat. Sci. Edition),2016,35(03):92-96.

[7] Sui Xiu-wu, YU Bao-fu, GE Hui, TIAN Song.Remote Intelligent Monitoring Strategy of Heat-Supply Network Based on Kingview[J].JOURNAL OF APPLIED SCIENCES-Electronics and Information Engineering,2016,34(03):352-360.

[8] Liu Qi,WANG Li,HOU Jie.Intelligent Regulation System of Indoor Heating[J].PROCESS AUTOMATION INSTRUMENTATION,2016,37(09):90-94.

[9] Miao Qing.Research on Application of Quantization Management and Intelligent Control for Central Heating System[D].Beijing University of Civil Engineering and Architecture,2016.

[10] Liu Yong Qi.Intelligent Control Algorithm Optimization in the Heat Transfer Station and Remote Monitoring System Design[D].Maritime Affairs University Of Dalian,2016. 